\title{
On Dimension Extension of a Class of Iterative Equations
}

\author{
Juan Su and Xinhan Dong \\ College of Mathematics and Computer Science, Hunan Normal University, Changsha 410081, China
}

Correspondence should be addressed to Juan Su; suj1234@126.com

Received 31 January 2013; Accepted 13 March 2013

Academic Editor: Chuangxia Huang

Copyright (C) 2013 J. Su and X. Dong. This is an open access article distributed under the Creative Commons Attribution License, which permits unrestricted use, distribution, and reproduction in any medium, provided the original work is properly cited.

\begin{abstract}
This investigation aims at studying some special properties (convergence, polynomial preservation order, and orthogonal symmetry) of a class of $r$-dimension iterative equations, whose state variables are described by the following nonlinear iterative equation: $\phi^{n}(x)=T\left(\phi^{n-1}(x)\right):=\sum_{j=0}^{m} H_{j} \phi^{n-1}(2 x-k)$. The obtained results in this paper are complementary to some published results. As an application, we construct orthogonal symmetric multiwavelet with additional vanishing moments. Two examples are also arranged to demonstrate the correctness and effectiveness of the main results.
\end{abstract}

\section{Introduction}

Giving any compact supported vector-valued function $\phi^{0}(x):=\left(\phi_{1}^{0}, \ldots, \phi_{r}^{0}\right)^{\top} \in\left(L^{2}(R)\right)^{r}$, we define $r$-dimension iterative equation as follows:

$$
\phi^{n}(x)=T\left(\phi^{n-1}(x)\right):=\sum_{j=0}^{\mu} H_{j} \phi^{n-1}(2 x-j),
$$

where $H_{j}$ is $r$-order real matrix, $j=0,1, \ldots, \mu, \mu \in Z^{+}$, and $n \in Z^{+}$. Let $H:=\left\{H_{j}, j=0,1, \ldots, \mu\right\}$ denote the masks of the iterative equation, then the Fourier transform of $\phi(x)=$ $\left(\phi_{1}, \ldots, \phi_{r}\right)^{\top}$, if it exists, can be defined by

$$
\widehat{\phi}(\omega)=\left(\int_{R} \phi_{1}(x) e^{-i \omega x} d x, \ldots, \int_{R} \phi_{r}(x) e^{-i \omega x} d x\right)^{\top},
$$

and the discrete-time Fourier transform (DTFT) of $H=\left\{H_{j}\right\}$ can be defined by

$$
\widehat{H}(\omega)=\frac{1}{2} \sum_{j=0}^{\mu} H_{j} e^{-i j \omega}
$$

where $i=\sqrt{-1}$. Therefore, iterative equation (1) takes the following frequency domain form:

$$
\widehat{\phi}^{n}(\omega)=\widehat{H}\left(\frac{\omega}{2}\right) \widehat{\phi}^{n-1}\left(\frac{\omega}{2}\right) .
$$

We claim that iterative equation (1) converges to a fixed function, and if $\lim _{n \rightarrow \infty} \phi^{n}(x)$ exists, we denote it by the $r$ dimension vector function $\phi(x):=\lim _{n \rightarrow \infty} \phi^{n}(x)$, whose frequency domain form is defined as an infinite product as follows:

$$
\widehat{\phi}(\omega)=\left[\prod_{k=1}^{\infty}\left[\widehat{H}\left(\frac{\omega}{2^{k}}\right)\right]\right] \widehat{\phi}(0) .
$$

Obviously, (5) is equivalent to $\phi(x)$ satisfying $r$-dimension refinement equation

$$
\phi(x)=\sum_{j=0}^{\mu} H_{j} \phi(2 x-j) .
$$

Iterative equation (1) is nonlinearity; in the real world, nonlinear problems are not exceptional, but regular phenomena. Nonlinearity is the nature of matter and its development $[1,2]$. Recently, iterative equation (1) has attracted increasing interest due to the potential applications in the field of wavelet analysis. In fact, the limit of iterative equation (1) satisfies refinement equation (6) which is fundamental to the theory of the scaling functions, and then we can construct special properties masks $H$ of iterative equation (1) to obtain scaling vector function with special properties. For example, in order to construct the wavelet frames with high order vanishing moments, in [3], Han and Mo investigate the method to factorize a matrix mask of (1). In order to construct multiwavelets with vanishing moments of arbitrarily high order, 
in [4], with the help of dimension extension and iterative scheme for revising masks of (1), Chui and Lian investigate the compactly supported orthogonal scaling function with additional polynomial preservation order (p.p.o.).

As we all know, the orthogonal symmetric scaling vector function with high p.p.o. is very important to construct symmetric multiwavelets with high vanishing moments by multiresolution analysis, and yet its construction is very difficult, especially in high dimension. The main objective of this paper is to develop an iterative equation to generate orthogonal symmetric scaling vector function as the limit of (1) and to analysis its convergence. Furthermore, we will introduce an iterative scheme by extending the dimension of iterative equation to obtain orthogonal symmetric scaling function with p.p.o increasing. As an application, we will construct compactly supported orthogonal symmetric multiwavelets to achieve any order vanishing moments.

\section{Preliminaries}

Just as shown in $[5,6]$, iterative equation (1) converges to a fixed point $\phi(x)$ or that the refinement equation (6) exists solution $\phi(x)$ if and only if matrix $\widehat{H}(0)$ satisfies Condition $E$ (for a matrix $A$, one says that $A$ satisfies Condition $E$ if the spectral radius of $A$ is equal to 1 , where 1 is the unique eigenvalue of $A$ on the unit circle, and it is a simple eigenvalue). The limit $\phi$ of (1) is called $r$-scaling vector function if $\phi$ is $L^{2}$-stable, meaning that $\left\{\phi_{l}(x-k): 1 \leq l \leq\right.$ $r$; $k \in Z\}$ is a Riesz basis of $V_{0}$, where, for $j \in Z$,

$$
V_{j}:=\operatorname{Clos}_{L^{2}} \operatorname{Span}\left\{2^{j / 2} \phi_{l}\left(2^{j} x-k\right): 1 \leq l \leq r ; k \in Z\right\},
$$

which is also called multiresolution analysis (MRA) of $L^{2}$, provided that $L^{2}=\operatorname{Clos}_{L^{2}} \bigcup_{j \in Z} V_{j}$.

According to $[6,7]$, iterative equation (1) converges to an $r$-dimension scaling vector function $\phi(x)$ if and only if the matrix $\widehat{H}(0)$ and the matrix $T_{H}$ satisfy Condition $E$, where, $T_{H}:=\left(A_{2 i-j}\right)_{1-\mu \leq i, j \leq \mu-1}, A_{j}$ is the $r^{2} \times r^{2}$ matrix given by $A_{j}:=$ $\sum_{k=0}^{\mu} H_{k-j} \otimes H_{k}$, and “ $\otimes$ " denotes the Kronecker product, that is, for two matrices $B=\left(b_{i j}\right)$ and $C=\left(c_{i j}\right), B \otimes C:=\left(b_{i j} C\right)$.

Definition 1 (see [4]). Let $\phi(x)=\left(\phi_{1}(x), \ldots, \phi_{r}(x)\right)^{\top}$ be $r$ dimension scaling function, and if polynomial $x^{l} \in V_{0}, l=$ $0,1, \ldots, m-1$, where $V_{0}:=\operatorname{Clos}_{L^{2}} \operatorname{Span}\left\{\phi_{l}(x-k): 1 \leq l \leq\right.$ $r ; k \in Z$, then we get that $\phi(x)$ has polynomial preservation order (p.p.o.)m.

Polynomial preservation order is a desired feature to a scaling function in application, for example, to construct multiwavelet with high-order vanishing moments. In [8], Plonka studied the polynomial preservation order properties of refinable function vectors in detail. Lian, in [9], established certain necessary and sufficient conditions for a multiscaling function $\phi(x)$ with p.p.o. $m$ in terms of the eigenvalues and their corresponding eigenvectors of masks of (1). Iterative equation (1) generates $r$-dimension scaling function $\phi(x)$ with p.p.o. $m$, if and only if the matrix masks of (1) satisfy $m$ order sum rules; that is, there exists real vector $y_{0}, y_{1}, \ldots, y_{m-1} \in R^{r}$ with $y_{0} \neq 0$, for any $0 \leq l \leq m-1$ such that

$$
\begin{gathered}
\sum_{k=0}^{l}(-1)^{k} \frac{1}{2^{k}}\left(\begin{array}{l}
l \\
k
\end{array}\right) y_{l-k} \sum_{j \in Z}(2 j)^{k} H_{2 j}=\frac{1}{2^{l}} y_{l}, \\
\sum_{k=0}^{l}(-1)^{k} \frac{1}{2^{k}}\left(\begin{array}{l}
l \\
k
\end{array}\right) y_{l-k} \sum_{j \in Z}(2 j+1)^{k} H_{2 j+1}=\frac{1}{2^{l}} y_{l},
\end{gathered}
$$

where $\left(\begin{array}{l}l \\ k\end{array}\right)=l ! / k !(l-k) !$.

A function vector $\eta=\left(\eta_{1}, \ldots, \eta_{r}\right)^{\top}$ is said to be orthogonal if it satisfies $\left\langle\eta_{l}(\cdot-k), \eta_{i}(\cdot-\hat{k})\right\rangle=\delta_{l, l} \delta_{k, k}, l, l=$ $1, \ldots, r ; k, \hat{k} \in Z$. If $\phi(x)$ satisfying refinement equation is orthogonal scaling function vector, then the masks of (1) must satisfy condition

$$
\widehat{H}(\omega) \widehat{H}^{\top}(-\omega)+\widehat{H}(\omega+\pi) \widehat{H}^{\top}(-\omega-\pi)=I_{r} .
$$

A scaling function vector $\phi(x)$ has symmetry property if all of its components are either symmetric or antisymmetric. The symmetry of $\phi$ is decided by the masks of (1). From [4], let $\phi$ be symmetric scaling vector function generated by (1) with two-scale symbol $H(z):=(1 / 2) \sum_{j=0}^{\mu} H_{k} z^{j}, z=e^{-i \omega}$ if and only if (see [4])

$$
S_{0} H(z) S_{0}=D_{0}\left(z^{2}\right) H\left(z^{-1}\right) D_{0}\left(z^{-1}\right),
$$

where $S_{0}=\operatorname{diag}\left((-1)^{i_{1}}, \ldots,(-1)^{i_{r}}\right), D_{0}(z):=\operatorname{diag}\left(z^{a_{1}+b_{1}}\right.$, $\left.\ldots, z^{a_{r}+b_{r}}\right)$ with supp $\phi_{l}=\left[a_{l}, b_{l}\right], l=1, \ldots, r ; i_{1}, \ldots, i_{r}$ being either 0 or 1 , depending on symmetry or antisymmetry of the corresponding components of $\phi$, respectively.

Let $\phi$ be $r$-dimension orthogonal symmetric scaling function which satisfies (6) with two-scale symbol $H(z)$ satisfying

$$
z^{2 \gamma-1} S_{0} H\left(z^{-1}\right) S_{0}=H(z)
$$

where $S_{0}=\operatorname{diag}\left(I_{s},-I_{r-s}\right)$, for a nonnegative integer $0<$ $s<r, \gamma \in Z^{+}$. In this case, if $H(z)$ satisfies (11) and (1) generates orthogonal symmetric scaling function vectors $\phi=$ $\left(\phi_{1}, \ldots, \phi_{r}\right)^{\top}$, then $\phi_{1}, \ldots, \phi_{s}$ are symmetric about $\gamma-(1 / 2)$ and $\phi_{s+1}, \ldots, \phi_{r}$ are antisymmetric about $\gamma-(1 / 2)$.

As shown in [10], the masks of (1) or two-scale symbol $H(z)$ of (6) satisfy (9) and (11) if and only if the following formulae hold:

$$
H(z)=\frac{\sqrt{2}}{2}\left[\begin{array}{cc}
a_{0} & 0 \\
0 & b_{0}
\end{array}\right] V_{1}\left(z^{2}\right) \cdots V_{\gamma-1}\left(z^{2}\right) U_{0}\left[\begin{array}{c}
I_{r} \\
I_{r} z
\end{array}\right],
$$

where $V_{j}(z)$ is defined by

$$
\begin{array}{r}
V_{i}(z):=\frac{1}{2}\left[\begin{array}{cc}
I_{r} & -v_{i} \\
-v_{i}^{\top} & I_{r}
\end{array}\right]+\frac{1}{2}\left[\begin{array}{cc}
I_{r} & v_{i} \\
v_{i}^{\top} & I_{r}
\end{array}\right] z, \quad v_{j} \in O(r), \\
j=1, \ldots, \gamma-1,
\end{array}
$$


where $a_{0}$ and $b_{0}$ are $s \times r$ and $(r-s) \times r$ matrices, respectively, $U_{0}=(\sqrt{2} / 2)\left[\begin{array}{cc}I_{r} & S_{0} \\ -I_{r} & S_{0}\end{array}\right]$.

If there exist matrices $a_{1}, b_{1}$, such that $\left[a_{0}^{\top}, a_{1}^{\top}\right],\left[b_{0}^{\top}, b_{1}^{\top}\right]$ being $r \times r$ orthogonal matrix, one can define $G(z)$ as follows:

$$
\begin{aligned}
G(z):= & \frac{1}{2} \sum_{k=0}^{2 \gamma-1} G_{k} z^{k}=\frac{\sqrt{2}}{2}\left[\begin{array}{cc}
0 & b_{1} \\
a_{1} & 0
\end{array}\right] V_{1}\left(z^{2}\right) \cdots V_{\gamma-1}\left(z^{2}\right) \\
& \times U_{0}\left[\begin{array}{c}
I_{r} \\
I_{r} z
\end{array}\right],
\end{aligned}
$$

and then we have

$$
\begin{gathered}
-z^{2 \gamma-1} S_{0} G\left(z^{-1}\right) S_{0}=G(z) ; \\
H(z) G^{\top}\left(z^{-1}\right)+H(-z) G^{\top}\left(-z^{-1}\right)=0, \quad z=e^{-i \omega} .
\end{gathered}
$$

Let $\phi(x)$ be $r$-dimension orthogonal symmetric scaling function satisfying refinement equation (6) with two-scale symbol $H(z)$ satisfying (11). If $G(z)$ is defined by (14), define $\psi(x):=$ $\left(\psi_{1}, \ldots, \psi_{r}\right)^{\top}$ by

$$
\psi(x):=\sum_{k=0}^{2 \gamma-1} G_{k}(2 x-k)
$$

and then $\psi(x)$ is $r$-dimension orthogonal symmetric multiwavelet function; that is, $\left\{2^{j / 2} \psi_{l}\left(2^{j} x-k\right) ; j, k \in Z, l=\right.$ $1,2, \ldots, r\}$ is the orthogonal basis of $L^{2}(R)$. When scaling function $\phi(x)$ has p.p.o. $=m$, one obtains that multi-wavelet function $\psi(x)$ has $m$ order vanishing moments; that is,

$$
\int_{R} x^{k} \psi_{l}(x) d x=0, \quad k=0,1, \ldots, m-1 ; l=1,2, \ldots, r .
$$

\section{Main Results}

At first, we give the following convergent lemma.

Lemma 2. Iterative equation (1) with any given compact supported vector-valued function $\phi^{0}(x)$ converges to a unique vector function $\phi$, if and only if the spectral radius of $\widehat{H}(0)$ is equal to 1,1 is the unique eigenvalue of $\widehat{H}(0)$ on the unit circle, and 1 is simple.

Proof. Using Fourier transform, from (1), (4), and (5), we obtain that the iterative equation (1) converges to vector function $\phi(x)$ if and only if the infinite product (5) converges. From [11], it is equivalent to the spectral radius of $\widehat{H}(0)$ which is equal to 1,1 is the unique eigenvalue of $\widehat{H}(0)$ on the unit circle, and 1 is simple. This completes the proof.

When iterative equations (1) with masks $H=\left\{H_{j}, j=\right.$ $0,1, \ldots, 2 \gamma-1\}$ generate $r$-dimension orthogonal symmetric scaling function $\phi(x)$ with p.p.o. $=m$, we can establish the following theorem to increase scaling function p.p.o. by extending the dimension of iterative equation (1).
Theorem 3. Let $\phi$ be $r$-dimension orthogonal symmetric scaling function with p.p.o. = m generated by iterative equation (1) with mask $H=\left\{H_{j}, j=0,1, \ldots, 2 \gamma-1\right\}$, where $H(z)$ satisfying (11), and Construct $r+2$-dimension iterative equations mask $\bar{H}=\left\{\bar{H}_{j}, j=0,1, \ldots, 2 \gamma-1\right\}$ as follows:

$$
\bar{H}(z):=\frac{1}{2} \sum_{j=0}^{2 \gamma-1} \bar{H}_{j} z^{j}=\left(\begin{array}{cc}
H(z) & 0_{r \times 2} \\
B G(z) & \frac{1+z^{2 \gamma-1}}{2^{m+1}} I_{2}
\end{array}\right),
$$

where $G(z)$ defined by (14). Then there exists $2 \times r$ matrix $B$ such that iterative equation (1) with mask $\bar{H}$ generates $(r+2)$-dimension orthogonal symmetric scaling function $\bar{\phi}=$ $\left(\phi^{\top}, \phi_{r+1}, \phi_{r+2}\right)^{\top}$ which has p.p.o. $\geq m+1$.

Proof. First, if matrix $H(1)$ satisfies the conditions of Lemma 2, then obviously, matrix $\bar{H}(1)$ constructed by (18) satisfies all conditions of Lemma 2. That is to say that the $r+2-$ dimension iterative equation (1) with mask $\bar{H}$ constructed by (18) converges to $\bar{\phi}$.

By applying the p.p.o. $=m$ of $\phi$ and sum rules of (8), there exist $y_{l}^{\top} \in R^{r}, l=0,1, \ldots, m-1$ with $y_{0} \neq 0_{1 \times r}$, for $0 \leq l \leq m-1$ satisfying

$$
\begin{gathered}
y_{l}\left(\sum_{j \in Z} H_{2 j}-\frac{1}{2^{l}}\right)=-\sum_{k=0}^{l-1}(-1)^{l-k} \frac{1}{2^{l-k}}\left(\begin{array}{l}
l \\
k
\end{array}\right) y_{k} \sum_{j \in Z}(2 j)^{l-k} H_{2 j}, \\
y_{l}\left(\sum_{j \in Z} H_{2 j+1}-\frac{1}{2^{l}}\right)= \\
\times \sum_{k=0}^{l-1}(-1)^{l-k} \frac{1}{2^{l-k}}\left(\begin{array}{l}
l \\
k
\end{array}\right) y_{k} \\
\times \sum_{j \in Z}(2 j+1)^{l-k} H_{2 j+1} .
\end{gathered}
$$

When $l=m$, there is no $r$-dimension row vector $y_{m}$ which satisfies (19). Now we will show that $\bar{H}(z)$ satisfies $m+1$ order sum rules by choosing matrix $B$.

Let $\bar{y}_{k}=\left(y_{k}, 0,0\right), k=0, \ldots, m-1, \bar{y}_{m}=\left(y_{m}, c_{m}^{1}, c_{m}^{2}\right)$, $c_{m}^{1}, c_{m}^{2} \in R, y_{m}$ being some $r$-dimension vector, then it is easy to obtain that $\bar{H}(z)$ satisfies $m$ order sum rules; that is for $0 \leq$ $l \leq m-1$, we have

$$
\begin{aligned}
\bar{y}_{l}\left(\sum_{j \in Z} \bar{H}_{2 j}-\frac{1}{2^{m}} I_{r}\right)= & -\sum_{k=0}^{l-1}(-1)^{l-k} \frac{1}{2^{l-k}}\left(\begin{array}{l}
l \\
k
\end{array}\right) \bar{y}_{k} \\
& \times \sum_{j \in Z}(2 j)^{l-k} \bar{H}_{2 j}, \\
\bar{y}_{l}\left(\sum_{j \in Z} \bar{H}_{2 j+1}-\frac{1}{2^{m}}\right)= & -\sum_{k=0}^{l-1}(-1)^{l-k} \frac{1}{2^{l-k}}\left(\begin{array}{l}
l \\
k
\end{array}\right) \bar{y}_{k} \\
& \times \sum_{j \in Z}(2 j+1)^{l-k} \bar{H}_{2 j+1} .
\end{aligned}
$$


When $l=m,(20)$ is equivalent to

$$
\begin{gathered}
y_{m}\left(\sum_{j \in Z} H_{2 j}-\frac{1}{2^{m}} I_{r}\right)+\left(c_{m}^{1}, c_{m}^{2}\right) B \sum_{j \in Z} G_{2 j} \\
=-\sum_{k=0}^{m-1}(-1)^{l-k} \frac{1}{2^{l-k}}\left(\begin{array}{l}
l \\
k
\end{array}\right) y_{k} \sum_{j \in Z}(2 j)^{l-k} H_{2 j}, \\
y_{m}\left(\sum_{j \in Z} H_{2 j+1}-\frac{1}{2^{m}}\right)+\left(c_{m}^{1}, c_{m}^{2}\right) B \sum_{j \in Z} G_{2 j+1} \\
=-\sum_{k=0}^{m-1}(-1)^{l-k} \frac{1}{2^{l-k}}\left(\begin{array}{l}
l \\
k
\end{array}\right) y_{k} \sum_{j \in Z}(2 j+1)^{l-k} H_{2 j+1} .
\end{gathered}
$$

When $\left(c_{m}^{1}, c_{m}^{2}\right)=(0,0)$, for $\phi$ with p.p.o. $=m$, there does not exist $y_{m}$ satisfying (21), but we will show that by choosing $2 \times r$ matrix $B$ and $\left(c_{m}^{1}, c_{m}^{2}\right)$, there exists $y_{m}$ satisfying (21), thus $\bar{H}(z)$ satisfies $m+1$ order sum rules. Let

$$
\begin{gathered}
p_{0}=:-\sum_{k=0}^{m-1}(-1)^{l-k} \frac{1}{2^{l-k}}\left(\begin{array}{l}
l \\
k
\end{array}\right) y_{k} \sum_{j \in Z}(2 j)^{l-k} H_{2 j}, \\
p_{1}=:-\sum_{k=0}^{m-1}(-1)^{l-k} \frac{1}{2^{l-k}}\left(\begin{array}{l}
l \\
k
\end{array}\right) y_{k} \sum_{j \in Z}(2 j+1)^{l-k} H_{2 j+1} .
\end{gathered}
$$

For $\phi$ with p.p.o. $=m,(19)$ is equivalent to the following equation (23) having no solutions:

$$
\left(\begin{array}{c}
\sum_{j \in Z} H_{2 j}^{\top}-\frac{1}{2^{m}} I_{r} \\
\sum_{j \in Z} H_{2 j+1}^{\top}-\frac{1}{2^{m}} I_{r}
\end{array}\right)\left(\begin{array}{c}
x_{1} \\
\vdots \\
x_{r}
\end{array}\right)=\left(\begin{array}{c}
p_{0}^{\top} \\
p_{1}^{\top}
\end{array}\right) .
$$

Because $H(z), G(z)$ are symmetric which satisfy (11) and (14), the following matrix is nonsingular:

$$
\left(\begin{array}{cc}
\sum_{j \in Z} H_{2 j}-\frac{1}{2^{m}} I_{r} & \sum_{j \in Z} H_{2 j+1}-\frac{1}{2^{m}} I_{r} \\
\sum_{j \in Z} G_{2 j} & \sum_{j \in Z} G_{2 j+1}
\end{array}\right) .
$$

We claim that the following system of linear equations (25) has solutions $\left(x_{1}, x_{2}, \ldots, x_{2 r}\right)^{\top}$ :

$$
\left(\begin{array}{cc}
\sum_{j \in Z} H_{2 j}^{\top}-\frac{1}{2^{m}} I_{r} & \sum_{j \in Z} G_{2 j+1}^{\top} \\
\sum_{j \in Z} H_{2 j+1}^{\top}-\frac{1}{2^{m}} I_{r} & \sum_{j \in Z} G_{2 j+1}^{\top}
\end{array}\right)\left(\begin{array}{c}
x_{1} \\
\vdots \\
x_{2 r}
\end{array}\right)=\left(\begin{array}{c}
p_{0}^{\top} \\
p_{1}^{\top}
\end{array}\right) .
$$

Let

where

$$
y_{m}=:\left(x_{1}, \ldots, x_{r}\right), \quad B=\left(\alpha_{1}^{\top}, \alpha_{2}^{\top}\right)^{\top},
$$

$$
\begin{aligned}
& \alpha_{1}=\sqrt{\frac{1-1 / 4^{m}}{x_{r+1}^{2}+\cdots+x_{r+s}^{2}}}\left(x_{r+1}, \ldots, x_{r+s}, 0_{1 \times r-s}\right), \\
& \alpha_{2}=\sqrt{\frac{1-1 / 4^{m}}{x_{r+s+1}^{2}+\cdots+x_{2 r}^{2}}}\left(0_{1 \times s}, x_{r+s+1}, \ldots, x_{2 r}\right) .
\end{aligned}
$$

Define

$$
c_{m}^{1}=\sqrt{\frac{x_{r+1}^{2}+\cdots+x_{r+s}^{2}}{1-1 / 4^{m}}}, \quad c_{m}^{2}=\sqrt{\frac{x_{r+s+1}^{2}+\cdots+x_{2 r}^{2}}{1-1 / 4^{m}}} .
$$

For (25), (26), and (27), we have that the row vectors $y_{l}, l=$ $0,1, \ldots, m,\left(c_{m}^{1}, c_{m}^{2}\right)$ and matrix $B$ satisfy $(21)$, that is to say that the scale symbol $\bar{H}(z)$ satisfies sum rules order $\geq m+1$.

In the following, we will show that $\bar{H}(z)$ is orthogonal symmetric two-scale symbol. For $H(z), G(z)$ satisfying (11), (14), and the defined matrix $B$, it is easy to obtain

$$
\begin{aligned}
& z^{2 N-1} \operatorname{diag}\left(s_{0},-1,1\right) \bar{H}\left(z^{-1}\right) \operatorname{diag}\left(s_{0},-1,1\right)=\bar{H}(z), \\
& \bar{H}(z) \bar{H}^{\top}\left(z^{-1}\right)+\bar{H}(-z) \bar{H}^{\top}\left(-z^{-1}\right)=\left(\begin{array}{cc}
I_{r} & 0_{r \times 2} \\
0_{2 \times r} & B B^{\top}+\frac{1}{4^{m}} I_{2}
\end{array}\right) \\
& =I_{r+2} \text {. }
\end{aligned}
$$

By (21) and (26)-(29), we have showed that $\bar{H}(z)$ is orthogonal symmetric two-scale symbol and satisfies at least $m+1$ order sum rules, that is to say that iterative function system (1) with mask $\bar{H}$ generates $r+2$-dimension orthogonal symmetric scaling function vector with p.p.o. $\geq m+1$. This completes the proof of Theorem 3.

By applying Theorem 3 to a pair of orthogonal symmetric scaling and wavelet vector functions, not only do we obtain a new scaling vector function with higher p.p.o., but also some corresponding orthogonal symmetric multi-wavelet vector function can be easily constructed. Precisely, we have the following.

Theorem 4. Let $\phi, \psi$ be r-dimension orthogonal symmetric scaling and wavelet function vectors with two-scale symbols $H(z), G(z)$ satisfying (11) and (14), respectively, where $\phi$ has p.p.o. $=m$, and then we can construct $\bar{H}(z)$ according to Theorem 3, and $r+2$-dimension corresponding two-scale symbol $\bar{G}(z)$ by

$$
\bar{G}(z)=\left(\begin{array}{cc}
A G(z) & -2^{m-1}\left(1+z^{2 \gamma-1}\right) A B^{\top} \\
0_{2 \times r} & \frac{-1+z^{2 \gamma-1}}{2} I_{2}
\end{array}\right),
$$

where $A=\operatorname{diag}\left(A_{1}, A_{2}\right), A_{1}, A_{2}$ being $s$ and $r-s$ order matrices, respectively, satisfies condition

$$
A A^{\top}+4^{m} A B^{\top} B A^{\top}=I_{r}
$$

Defining $\bar{\phi}=\left(\phi^{\top}, \phi_{r+1}, \phi_{r+2}\right)^{\top}, \bar{\psi}=\left(\bar{\psi}_{1}, \ldots, \bar{\psi}_{r+2}\right)^{\top}$ generated by $\bar{H}(z), \bar{G}(z)$, then $\bar{\phi}, \bar{\psi}$ are orthogonal symmetric, and $\bar{\psi}$ has at least $m+1$ vanishing moments.

Proof. First, we show that $\bar{G}(z)$ satisfies symmetry conditions. In Theorem 3 , we have proved that $\bar{H}(z)$ satisfies symmetric condition

$$
z^{2 \gamma-1} \operatorname{diag}\left(s_{0},-1,1\right) \bar{H}\left(z^{-1}\right) \operatorname{diag}\left(s_{0},-1,1\right)=\bar{H}(z) .
$$


For matrix $B$ given by (26) and (27), $A=\operatorname{diag}\left(A_{1}, A_{2}\right)$ with $A_{1}, A_{2}$ being $s$ and $r-s$ order matrices, respectively, and $G(z)$ satisfying (14), we have that $\bar{G}(z)$ satisfies symmetric condition

$$
z^{2 \gamma-1} \operatorname{diag}\left(-s_{0}, 1,-1\right) \bar{G}\left(z^{-1}\right) \operatorname{diag}\left(s_{0},-1,1\right)=\bar{G}(z) .
$$

$H(z), G(z)$ are orthogonal two-scale symbols, then it is easy to get

$$
\begin{array}{r}
\bar{G}(z) \bar{G}^{\top}\left(z^{-1}\right)+\bar{G}(-z) \bar{G}^{\top}\left(-z^{-1}\right) \\
=\left(\begin{array}{cc}
A A^{\top}+4^{m} A B^{\top} B A^{\top} & 0_{r \times 2} \\
0_{2 \times r} & I_{2}
\end{array}\right), \\
\bar{H}(z) \bar{G}^{\top}\left(z^{-1}\right)+\bar{H}(-z) \bar{G}^{\top}\left(-z^{-1}\right) \\
=\left(\begin{array}{cc}
0_{r \times r} & 0_{r \times 2} \\
B A^{\top}-B A^{\top} & 0_{2 \times 2}
\end{array}\right)=0_{r+2} .
\end{array}
$$

From (34), when condition (31) is satisfied, $\bar{H}(z)$ and $\bar{G}(z)$ constructed by Theorem 3 and Theorem 4 are orthogonal symmetric two-scale symbols and wavelet functions with at least $m+1$ order vanishing moments. This completes the proof of Theorem 4 .

One of the important features of the construction procedure described in Theorem 3 and Theorem 4 is that it can be applied repeatedly without increasing the support (or filter length). In Theorem 3, $2 \times r$ matrix $B$ is decided by twoscale symbol $H(z)$. In Theorem 4 , matrix $A=\operatorname{diag}\left(A_{1}, A_{2}\right)$ is constructed by matrix $B$ with condition (31). How can we obtain matrix $A=\operatorname{diag}\left(A_{1}, A_{2}\right)$ satisfying (31)? Considering matrix $B$ given by (26) and (27), we have the following theorem.

Theorem 5. Let $2 \times r$ matrix $B=\left(\begin{array}{cc}b_{1} & 0_{1 \times r-s} \\ 0_{1 \times s} & b_{2}\end{array}\right)$ be given by (26) and (27), and let matrix $A=\operatorname{diag}\left(A_{1}, A_{2}\right)$ satisfy (31) with $A_{1}, A_{2}$ being $s$ and $r-s$ order matrices, respectively, and then $A_{1}, A_{2}$ can be obtained by

$$
\begin{gathered}
A_{1}=\operatorname{diag}\left(\frac{1}{2^{m}}, I_{s-1}\right)\left(b_{1}^{0 \top}, v_{1}, \ldots, v_{s-1}\right)^{\top}, \\
A_{2}=\operatorname{diag}\left(\frac{1}{2^{m}}, I_{r-s-1}\right)\left(b_{2}^{0 \top}, \bar{v}_{1}, \ldots, \bar{v}_{r-s-1}\right)^{\top},
\end{gathered}
$$

where $b_{1}^{0}=\left(1 /\left|b_{1}\right|\right) b=\left(2^{m} / \sqrt{4^{m}-1}\right) b_{1}, v_{1}, \ldots, v_{s-1}$ are unit orthogonal complement vectors of $b_{1}^{0 \top}$, and $b_{2}^{0}=\left(1 /\left|b_{2}\right|\right) b_{2}=$ $\left(2^{m} / \sqrt{4^{m}-1}\right) b_{2}, \bar{v}_{1}, \ldots, \bar{v}_{r-s-1}$ are unit orthogonal complement vectors of $b_{2}^{0^{\top}}$.

Proof. Condition (31) is equivalent to

$$
I_{r}+4^{m} B^{\top} B=A^{-1}\left(A^{-1}\right)^{\top} .
$$

For the $A=\operatorname{diag}\left(A_{1}, A_{2}\right)$ and $B=\left(\begin{array}{cc}b_{1} & 0_{1 \times r-s} \\ 0_{1 \times s} & b_{2}\end{array}\right)$, we have

$$
I_{s}+4^{m} b_{1}^{\top} b_{1}=A_{1}^{-1}\left(A_{1}^{-1}\right) \top, \quad I_{r-s}+4^{m} b_{2}^{\top} b_{2}=A_{2}^{-1}\left(A_{2}^{-1}\right)^{\top} \text {. }
$$

Matrix $I_{s}+4^{m} b_{1}^{\top} b_{1}$ has eigenvalue $1+4^{m}\left|b_{1}\right|^{2}$ and 1. For (26) and (27), we have $1+4^{m}\left|b_{1}\right|^{2}=4^{m}$. Characteristic unit vectors $b_{1}^{0 \top}=(1 /|b|) b^{\top}$ correspond to eigenvalue $4^{m}$ and $v_{1}, \ldots, v_{s-1}$ which are orthogonal complement vectors of $b_{1}^{0 \top}$ and characteristic unit vectors correspond to eigenvalue 1 of matrix $I_{s}+4^{m} b^{\top} \mathrm{b}$, and then we can get

$$
\begin{aligned}
\left(I_{s}\right. & \left.+4^{m} b_{1}^{\top} b_{1}\right)\left(b_{1}^{0 \top}, v_{1}, \ldots, v_{s-1}\right) \\
& =\left(b_{1}^{0^{\top}}, v_{1}, \ldots, v_{s-1}\right) \operatorname{diag}\left(4^{m}, I_{s-1}\right), \\
I_{s} & 4^{m} b_{1}^{\top} b_{1} \\
& =\left(b_{1}^{0 \top}, v_{1}, \ldots, v_{s-1}\right) \operatorname{diag}\left(4^{m}, I_{s-1}\right)\left(b_{1}^{0^{\top}}, v_{1}, \ldots, v_{s-1}\right)^{\top} .
\end{aligned}
$$

From (37) and (38), we obtain

$$
A_{1}=\operatorname{diag}\left(\frac{1}{2^{m}}, I_{s-1}\right)\left(b_{1}^{0 \top}, v_{1}, \ldots, v_{s-1}\right)^{\top} .
$$

In the same way, we have

$$
A_{2}=\operatorname{diag}\left(\frac{1}{2^{m}}, I_{r-s-1}\right)\left(b_{2}^{0 \top}, \bar{v}_{1}, \ldots, \bar{v}_{r-s-1}\right)^{\top} .
$$

This completes the proof of Theorem 5 .

\section{Example}

Applying Theorems 3, 4, and 5, it is easy to extend the $r$ dimension orthogonal symmetric multi-wavelet to $r+2$ dimension orthogonal symmetric multi-wavelet with vanishing moments increasing. In Theorem 3 , if matrix $B$ is constructed by (27) with $\alpha_{1}=0_{1 \times r}$ or $\alpha_{2}=0_{1 \times r}$, we can extend the $r$-dimension orthogonal symmetric multi-wavelet to $r+1$-dimension orthogonal symmetric multi-wavelet with vanishing moments increasing without increasing the support of wavelet functions, and then we will give two examples to show it.

Example 6. Let

$$
\begin{gathered}
H(z)=\frac{1}{2}\left(\begin{array}{cc}
1+z & 0 \\
\frac{1}{2}-\frac{1}{2} z & -\frac{\sqrt{3}}{2}-\frac{\sqrt{3}}{2} z
\end{array}\right), \\
G(z)=\frac{1}{2}\left(\begin{array}{cc}
\frac{\sqrt{3}}{2}-\frac{\sqrt{3}}{2} z & \frac{1}{2}+\frac{1}{2} z \\
0 & 1-z
\end{array}\right)
\end{gathered}
$$

be two-scale symbols and satisfy

$$
\begin{gathered}
z \operatorname{diag}(1,-1) H\left(z^{-1}\right) \operatorname{diag}(1,-1)=H(z), \\
z \operatorname{diag}(-1,1) G\left(z^{-1}\right) \operatorname{diag}(1,-1),
\end{gathered}
$$

and then (1) generates orthogonal symmetric 2D scaling function $\phi$ with p.p.o. $=1$, and $y_{0}=(1,0)$. From Theorem 3 , 
let $\alpha_{1}=(\sqrt{3} / 2,0), \alpha_{2}=(0,0)$, and define $B=\alpha_{1}, A=$ $\operatorname{diag}(1 / 2,1)$, and then we obtain $\bar{H}(z), \bar{G}(z)$ as follows:

$$
\begin{gathered}
\bar{H}(z)=\frac{1}{2}\left(\begin{array}{ccc}
1+z & 0 & 0 \\
\frac{1}{2}-\frac{1}{2} z & -\frac{\sqrt{3}}{2}-\frac{\sqrt{3}}{2} z & 0 \\
\frac{\sqrt{3}}{2}(\sqrt{3}-\sqrt{3}) z & \frac{\sqrt{3}}{2}(1+z) & \frac{1+z}{4}
\end{array}\right), \\
\bar{G}(z)=\frac{1}{2}\left(\begin{array}{ccc}
-\frac{\sqrt{3}}{4}+\frac{\sqrt{3}}{4} z & -\frac{1}{4}-\frac{1}{4} z & \frac{\sqrt{15}}{4}(1+z) \\
0 & 1-z & 0 \\
0 & 0 & -1+z
\end{array}\right) .
\end{gathered}
$$

Two-scale symbols $\bar{H}(z), \bar{G}(z)$ generate $3 \mathrm{D}$ scaling and wavelet function vectors $\bar{\phi}=\left(\phi_{1}, \phi_{2}, \phi_{3}\right)^{\top}$ with p.p.o. $=2$, $\bar{\psi}=\left(\psi_{1}, \psi_{2}, \psi_{3}\right)^{\top}$ with vanishing moment with order 2 , and $\phi_{1}, \psi_{2}, \psi_{3}$ symmetric, $\phi_{2}, \phi_{3}, \psi_{1}$ antisymmetric. $\bar{H}(z)$ satisfies sum rules with order 2 with $y_{0}=(1,0,0), y_{1}=$ $(1 / 2,-(1 / 2(\sqrt{3}+2)),-((\sqrt{3}+1) / 3(\sqrt{3}+2)))$.

Example 7. Let $\phi$ with p.p.o. $=3, \psi$ be $2 \mathrm{D}$ orthogonal symmetric scaling and wavelet function, respectively, generated by two-scale symbols [4]

$$
\begin{aligned}
& H(z)=\left(\begin{array}{ll}
H_{11}(z) & H_{12}(z) \\
H_{21}(z) & H_{22}(z)
\end{array}\right) \\
& G(z)=\left(\begin{array}{ll}
G_{11}(z) & G_{12}(z) \\
G_{21}(z) & G_{22}(z)
\end{array}\right)
\end{aligned}
$$

where

$$
\begin{aligned}
H_{11}(z)= & \frac{10-3 \sqrt{10}}{80}(1+z)\left(1+(38+12 \sqrt{10}) z+z^{2}\right), \\
H_{21}(z)= & \frac{5 \sqrt{6}-3 \sqrt{15}}{80}(1-z)\left(1-10(3+\sqrt{10}) z+z^{2}\right), \\
& H_{12}(z)=\frac{5 \sqrt{6}-2 \sqrt{15}}{80}(1-z)(1+z)^{2}, \\
H_{22}(z)= & \frac{5-3 \sqrt{10}}{1040}(1+z)\left(13-(10+6 \sqrt{10}) z+13 z^{2}\right), \\
& G_{11}(z)=\frac{5 \sqrt{6}-2 \sqrt{15}}{80}(1-z)^{2}(1+z), \\
G_{21}(z)= & -\frac{5-3 \sqrt{10}}{1040}(1-z)\left(13+(10+6 \sqrt{10}) z+13 z^{2}\right), \\
G_{12}(z)= & -\frac{10-3 \sqrt{10}}{80}(1-z)\left(1-(38+12 \sqrt{10}) z+z^{2}\right), \\
G_{22}(z)= & \frac{5 \sqrt{6}-3 \sqrt{15}}{80}(1+z)\left(1+10(3+\sqrt{10}) z+z^{2}\right),
\end{aligned}
$$

which satisfy

$$
\begin{gathered}
z^{3} \operatorname{diag}(1,-1) H\left(z^{-1}\right) \operatorname{diag}(1,-1)=H(z), \\
z^{3} \operatorname{diag}(-1,1) G\left(z^{-1}\right) \operatorname{diag}(1,-1) .
\end{gathered}
$$

By applying Theorems 3-5, we obtain a new pair of orthogonal scaling function $\bar{\phi}$ with p.p.o. $=4$ and multi-wavelet $\bar{\psi}$ that can be obtained from two-scale symbols $\bar{H}(z), \bar{G}(z)$ given by

$$
\begin{aligned}
& \bar{H}(z)=\left(\begin{array}{cc}
H(z) & 0_{2 \times 1} \\
B G(z) & \frac{1+z^{3}}{16}
\end{array}\right), \\
& \bar{G}(z)=\left(\begin{array}{cc}
A G(z) & \frac{1+z^{3}}{2} \xi \\
0_{1 \times 2} & \frac{1-z^{3}}{2}
\end{array}\right),
\end{aligned}
$$

where

$$
\begin{gathered}
B=\left(0, \frac{3 \sqrt{7}}{8}\right), \quad A=\operatorname{diag}\left(\frac{1}{8}, 1\right), \\
\xi=\left(-\frac{3 \sqrt{7}}{8}, 0\right)^{\top} .
\end{gathered}
$$

In addition, $\bar{H}(z)$ satisfies sum rules with order 4 with the four vectors given by

$$
\begin{gathered}
y_{0}=[1,0,0], \quad y_{1}=\left[\frac{3}{2}, \frac{\sqrt{15}-\sqrt{6}}{6}, 0\right], \\
y_{2}=\left[\frac{17-\sqrt{10}}{6}, \frac{\sqrt{15}-\sqrt{6}}{2}, 0\right], \\
y_{3}=\left[\frac{3(8-\sqrt{10})}{4}, \frac{\sqrt{3}(1280 \sqrt{5}-1373)}{996}, \frac{\sqrt{7}(9 \sqrt{10}-35)}{581}\right] .
\end{gathered}
$$

\section{Acknowledgments}

This study is supported by the Natural Sciences Foundation of China (11171100) and the Scientific Research Found of Hunan Provincial Education Department (no. 11W012).

\section{References}

[1] F. Wen and X. Yang, "Skewness of return distribution and coefficient of risk premium," Journal of Systems Science \& Complexity, vol. 22, no. 3, pp. 360-371, 2009.

[2] F. Wen, Z. Li, C. Xie, and S. David, "Study on the fractal and chaotic features of the Shanghai composite index," FractalsComplex Geometry Patterns and Scaling in Nature and Society, vol. 20, pp. 133-140, 2012.

[3] B. Han and Q. Mo, "Multiwavelet frames from refinable function vectors," Advances in Computational Mathematics, vol. 18, no. 2-4, pp. 211-245, 2003, Frames. 
[4] C. K. Chui and J. Lian, "Construction of orthonormal multiwavelets with additional vanishing moments," Advances in Computational Mathematics, vol. 24, no. 1-4, pp. 239-262, 2006.

[5] G. Plonka, "Necessary and Sufficient Conditions for Orthonormality of Scaling Vectors," Tech. Rep., University Rostock, 1997.

[6] W. M. Lawton, "Necessary and sufficient conditions for constructing orthonormal wavelet bases," Journal of Mathematical Physics, vol. 32, no. 1, pp. 57-61, 1991.

[7] Q. Jiang, "On the design of multifilter banks and orthonromal multiwavelet bases," IEEE Transactions on Signal Processing, vol. 46, pp. 3292-3303, 1998.

[8] G. Plonka, "Approximation order provided by refinable function vectors," Constructive Approximation, vol. 13, no. 2, pp. 221244, 1997.

[9] J.-A. Lian, "On the order of polynomial reproduction for multi-scaling functions," Applied and Computational Harmonic Analysis, vol. 3, no. 4, pp. 358-365, 1996.

[10] Q. Jiang, "Symmetric paraunitary matrix extension and parametrization of symmetric orthogonal multifilter banks," SIAM Journal on Matrix Analysis and Applications, vol. 23, no. 1, pp. 167-186, 2001.

[11] Z. Shen, "Refinable function vectors," SIAM Journal on Mathematical Analysis, vol. 29, no. 1, pp. 235-250, 1998. 


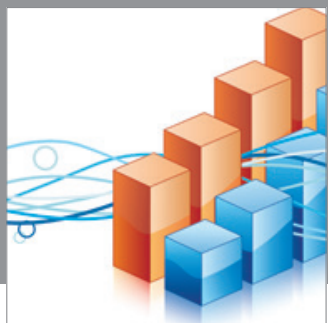

Advances in

Operations Research

mansans

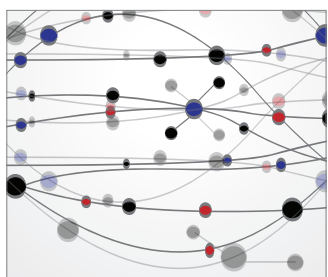

The Scientific World Journal
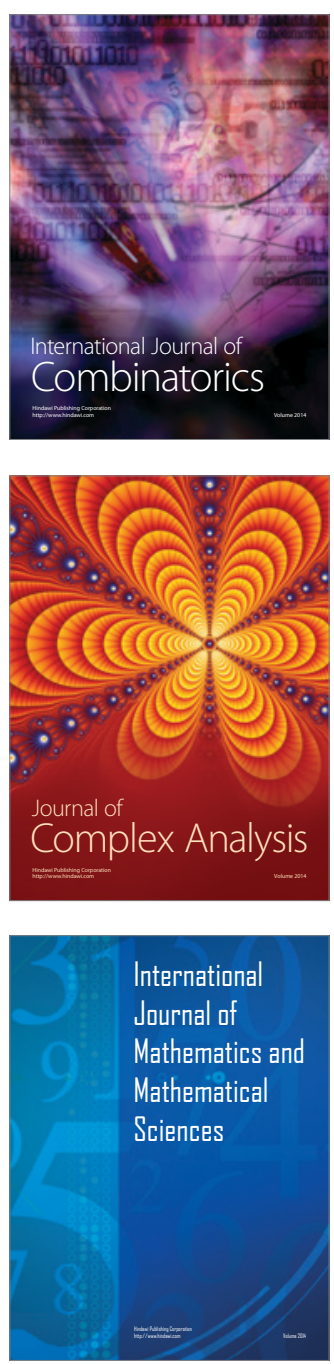
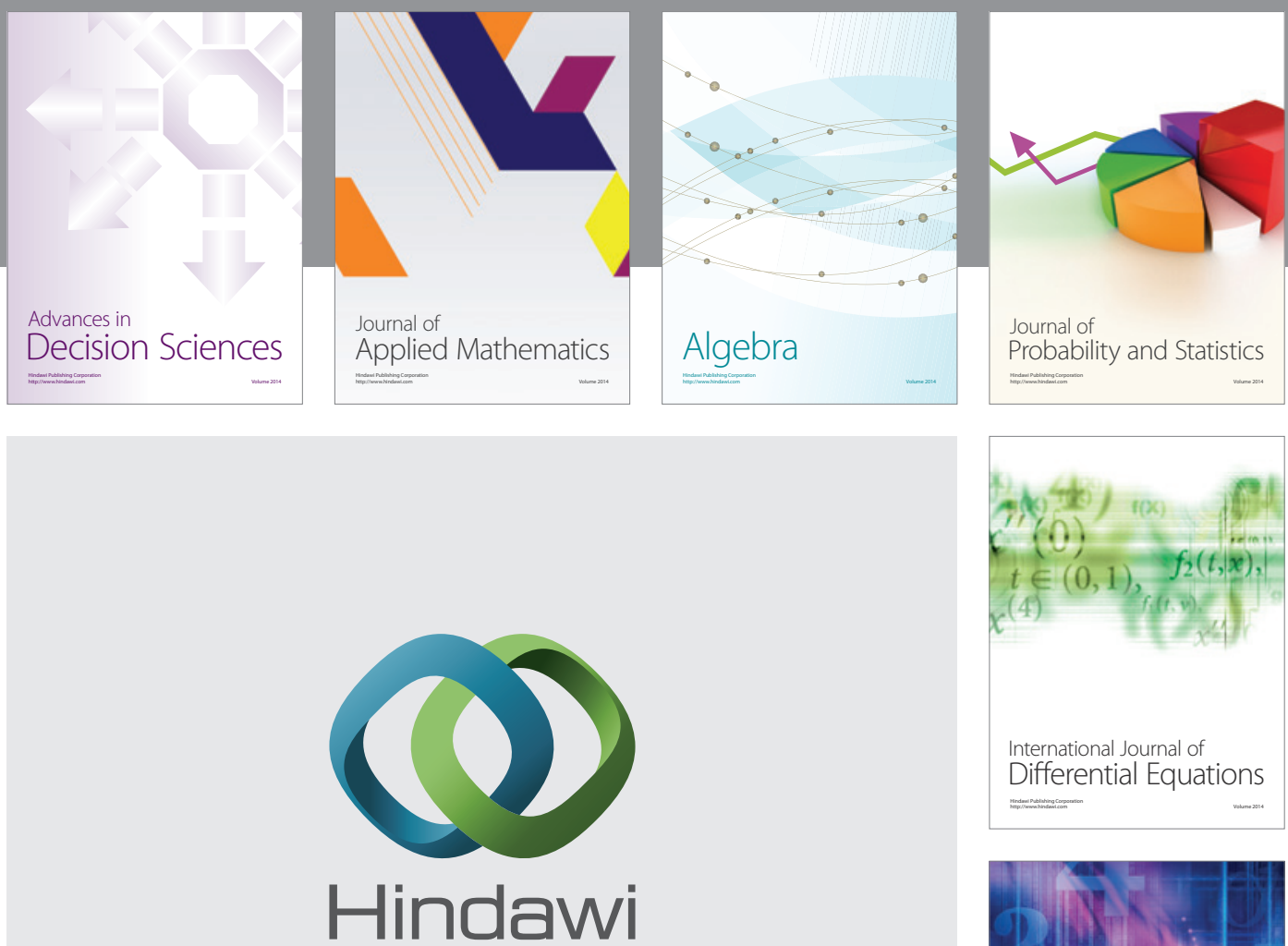

Submit your manuscripts at http://www.hindawi.com
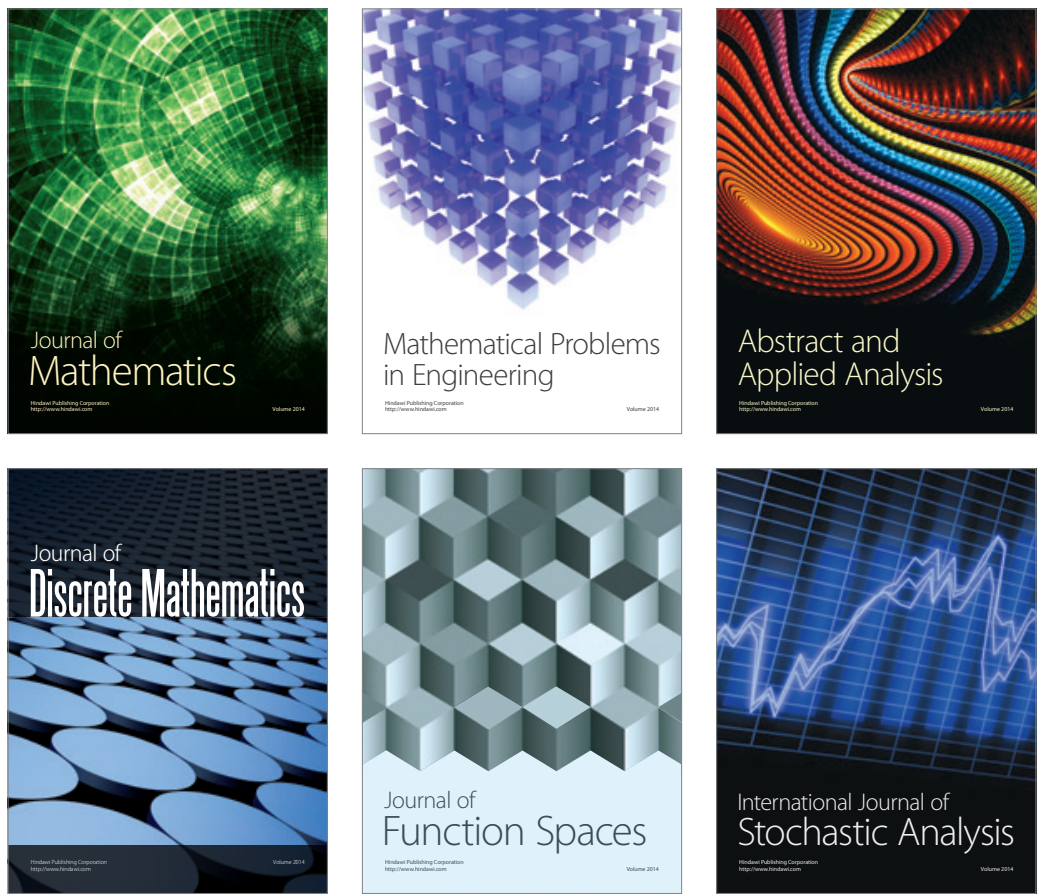

Journal of

Function Spaces

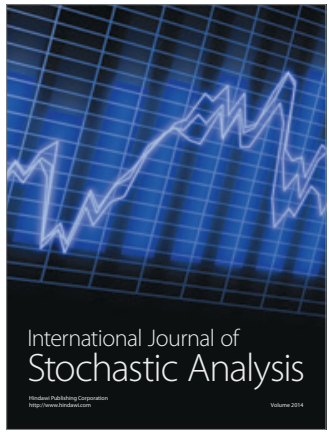

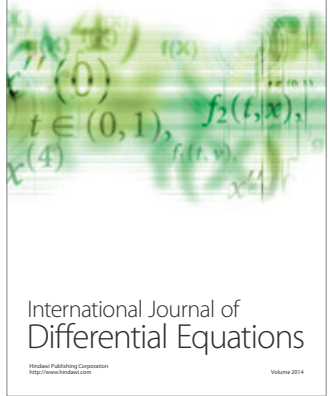
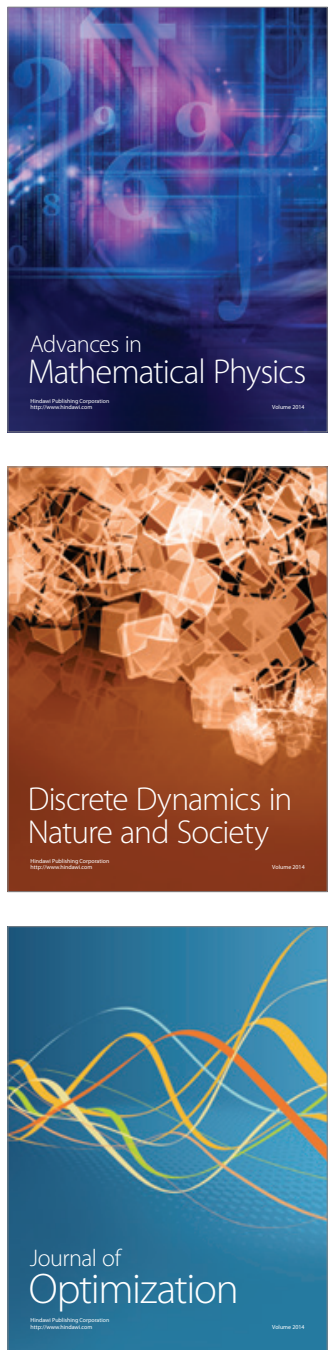